\title{
BMJ Open Protocol for a randomised controlled trial of VAsopressin versus Noradrenaline as Initial therapy in Septic sHock (VANISH)
}

\author{
Anthony C Gordon, ${ }^{1,2}$ Alexina J Mason, ${ }^{3}$ Gavin D Perkins, ${ }^{4,5}$ Deborah Ashby, ${ }^{3}$ \\ Stephen J Brett ${ }^{2}$
}

To cite: Gordon AC, Mason AJ, Perkins GD, et al. Protocol for a randomised controlled trial of VAsopressin versus Noradrenaline as Initial therapy in Septic sHock (VANISH). BMJ Open 2014;4: e005866. doi:10.1136/ bmjopen-2014-005866

- Prepublication history for this paper is available online. To view these files please visit the journal online (http://dx.doi.org/10.1136/ bmjopen-2014-005866).

Received 5 June 2014 Accepted 13 June 2014

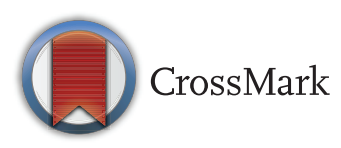

For numbered affiliations see end of article.

Correspondence to Dr Anthony C Gordon; anthony.gordon@imperial.ac. uk

\section{ABSTRACT}

Introduction: Vasopressin is an alternative vasopressor in the management of septic shock. It spares catecholamine use but whether it improves outcome remains uncertain. Current evidence suggests that it may be most effective if used early and possibly in conjunction with corticosteroids. This trial will compare vasopressin to noradrenaline as initial vasopressor in the management of adult septic shock and investigate whether there is an interaction of vasopressin with corticosteroids.

Methods and analysis: This is a multicentre, factorial $(2 \times 2)$, randomised, double-blind, placebocontrolled trial. 412 patients will be recruited from multiple UK intensive care units and randomised to receive vasopressin $(0-0.06 \mathrm{U} / \mathrm{min})$ or noradrenaline $(0-12 \mu \mathrm{g} / \mathrm{min})$ as a continuous intravenous infusion as initial vasopressor therapy. If maximum infusion rates of this first study drug are reached, the patient will be treated with either hydrocortisone (initially $50 \mathrm{mg}$ intravenous bolus six-hourly) or placebo, before additional open-label catecholamine vasopressors are prescribed. The primary outcome of the trial will be the difference in renal failure-free days between treatment groups. Secondary outcomes include need for renal replacement therapy, survival rates, other organ failures and resource utilisation.

Ethics and dissemination: The trial protocol and information sheets have received a favourable opinion from the Oxford A Research Ethics Committee (12/SC/ 0014). There is an independent Data Monitoring and Ethics Committee and independent membership of the Trial Steering Committee including patient and public involvement. The trial results will be published in peerreviewed journals and presented at national and international scientific meetings.

Trial registration number: ISRCTN 20769191 and EudraCT 2011-005363-24.

\section{INTRODUCTION}

Severe sepsis is an increasingly common problem worldwide and is an important cause of mortality (10th most common cause of death in the $\mathrm{USA}^{1}$ ). In the UK, the

\section{Strengths and limitations of this study}

Multicentre double-blind randomised trial.

- Comparing vasopressin and noradrenaline as early therapy in adult septic shock to prevent and reduce renal dysfunction.

- There is no long-term follow-up.

incidence of severe sepsis has increased $68 \%$ over a 9-year period, such that in 2004 there were 31000 patients who had severe sepsis admitted to intensive care units (ICUs) in England, Wales and Northern Ireland. ${ }^{2} \mathrm{~A}$ substantial number of patients will also develop severe sepsis after admission to intensive care, ${ }^{3}$ so the total number of severe sepsis cases may be in excess of $45000 /$ annum. As the population ages and receives more complex medical treatments, this will continue to rise.

Mortality from sepsis is $30-50 \%$, proportional to illness severity and the number of organs failing. Septic shock, the most severe form of sepsis, is defined as hypotension in response to overwhelming infection. ${ }^{4}$ As well as appropriate antibiotic treatment, one of the main treatments is cardiovascular resuscitation using intravenous fluids and catecholamines. Although usually effective in restoring blood pressure, catecholamines have important adverse effects and may even increase mortality. ${ }^{5}$

Vasopressin, an endogenous stress hormone, has been proposed as an adjunct to catecholamines in the treatment of septic shock. The rationale for its use is that a relative vasopressin deficiency occurs in septic shock and exogenously administered vasopressin restores vascular tone,${ }^{6}$ increases blood pressure and thus leads to a reduced requirement for catecholamine vasopressors. ${ }^{7}$ 
Furthermore, vasopressin exerts its effects via interaction with a family of vasopressin receptors. V1a receptors are located on vascular smooth muscle cells and are responsible for vasoconstriction. Heterogeneity of the distribution of V1a receptor could have important clinical and therapeutic implications. For example, vasopressin causes renal efferent, but not renal afferent, artery vasoconstriction thereby increasing renal perfusion pressure and glomerular filtration rate. ${ }^{8}$ In contrast, noradrenaline interacts with $\alpha-1$ receptors on renal afferent and efferent arterioles and so noradrenaline at high doses may decrease renal perfusion and glomerular filtration rate. ${ }^{9}$ Thus, in contrast to noradrenaline, there is a compelling organ-specific heterogeneity in the vascular responsiveness to vasopressin. Importantly, vasopressin also binds to oxytocin receptors, which, in addition to their uterine contractile effects, mediate calcium-dependent vasodilation via stimulation of the nitric oxide pathway in endothelial cells ${ }^{10}$ of pulmonary, ${ }^{11}$ coronary ${ }^{12}$ and cerebral arteries. ${ }^{13} 14$

There have been two recent meta-analyses of vasopressin (and its synthetic analogue terlipressin) in septic shock published that clearly demonstrated the catecholamine sparing effect of vasopressin and also suggested that its use appears safe. ${ }^{15} 16$ The effect on short-term mortality was not so clear. Serpa Neto et $a l,{ }^{15}$ using a fixed-effect model, reported a relative risk (RR) of death in adult patients treated with vasopressin/terlipressin of 0.87 (95\% CI 0.77 to 0.99 ). Polito et $a l,^{16}$ using a random-effect model, reported an RR for vasopressin/ terlipressin of 0.91 (95\% CI 0.79 to 1.05 ). The uncertainty about the effect of vasopressin on survival rates in septic shock is reflected in the 2008 international Surviving Sepsis guidelines ${ }^{17}$ that stated "Vasopressin... may be added to noradrenaline subsequently with anticipation of an effect equivalent to that of noradrenaline alone."

Therefore, there appears to be equipoise about the effect of vasopressin on outcome in septic shock and more trials are warranted. In order to design future trials, it is important to consider the results of the Vasopressin and Septic Shock Trial (VASST), the largest randomised controlled trial of vasopressin until now. ${ }^{18}$ In this multicentre trial, 779 patients who had established septic shock were randomised to receive a blinded infusion of either vasopressin or noradrenaline in addition to conventional catecholamine vasopressors. There was no evidence of a difference in 28-day mortality between the treatment groups $(35.4 \%$ vasopressin group and $39.3 \%$ noradrenaline group, $\mathrm{p}=0.26$ ) or in 90 -day mortality $(43.9 \%$ and $49.6 \%$, respectively, $\mathrm{p}=0.11)$. Importantly, vasopressin infusion in septic shock appeared safe. The overall serious adverse event (SAE) rate was the same in the vasopressin and noradrenaline groups $(10.3 \%$ and $10.5 \%$, respectively), and there was no difference in specific adverse events (AEs) including myocardial ischaemia $^{19}$ or cardiac output. ${ }^{20}$ However, in the predefined stratum of less severe shock, there was a reduced mortality in the vasopressin group compared with the noradrenaline group ( $26.5 \%$ vs $35.7 \%$ 28-day mortality, respectively, $\mathrm{p}=0.05$ ). There was no difference in mortality between treatment groups in patients who had more severe shock. In a post hoc subgroup analysis, the patient group treated with vasopressin within $12 \mathrm{~h}(\mathrm{n}=427)$ had a reduced mortality $(33.2 \%$ vs $40.5 \%, \mathrm{p}=0.12)$, but no difference in mortality if treated with vasopressin after $12 \mathrm{~h}$. In additional post hoc analyses, vasopressin treatment led to lower rates of progression to renal failure $(21.2 \%$ vs $41.2 \%, \mathrm{p}=0.02)$ and mortality $(30.8 \%$ vs $54.7 \%, \mathrm{p}=0.01$ ) in patients at 'Risk' of renal failure, but no difference in outcomes if renal failure was already established. $^{21}$ The Editorial accompanying the VASST report suggested that early use of vasopressin may be needed to produce a significant improvement in survival. $^{22}$

Another interesting subgroup analysis from VASST ${ }^{23}$ provided strong evidence of an interaction between vasopres$\sin /$ noradrenaline treatment and corticosteroid treatment (interaction $\mathrm{p}=0.008$ ). The combination of vasopressin and steroids led to a lower mortality compared with noradrenaline plus steroids ( $35.9 \%$ vs $44.7 \%$, respectively, $\mathrm{p}=0.03$ ) and less organ dysfunction demonstrated by more days alive and free from shock, ventilation and renal failure. In contrast, patients who were treated with vasopressin and had no corticosteroids had an increased mortality compared with patients who were treated with noradrenaline and no steroids ( $33.7 \%$ vs $21.3 \%$, respectively, $\mathrm{p}=0.06$ ). The use of corticosteroids in septic shock remains controversial despite more than 40 years of research. There is good evidence that they spare catecholamine requirements ${ }^{24}$ and recent meta-analyses reported a beneficial effect of prolonged low-dose corticosteroids in the more severely ill patients. $^{25} 26$

Therefore, in designing a further vasopressin trial in septic shock, we decided it was essential to start vasopressin administration early after the onset of hypotension and that we should control for a potential interaction of vasopressin with corticosteroids. Before starting this main trial, we conducted a pilot study, Vasopressin and Corticosteroids in Septic Shock (VACS) ${ }^{27}$ This randomised controlled trial demonstrated that it was possible to use vasopressin (up to $0.06 \mathrm{U} / \mathrm{min}$ ) safely as an early vasopressor and that there was evidence of a clinical interaction between vasopressin and corticosteroids. Patients randomised to vasopressin and corticosteroids required three fewer days of vasopressin treatment and also received less than half the total vasopressin dose compared with patients randomised to vasopressin and placebo. ${ }^{27}$

This protocol describes the main trial that compares vasopressin to noradrenaline as initial vasopressor in the management of adult septic shock. As there may be an interaction of vasopressin with corticosteroids, the use of corticosteroids will also be controlled but only given to patients who are poorly responsive to fluids and vasopressors, according to international guidelines. ${ }^{17}$ 


\section{METHODS AND ANALYSIS}

The VAsopressin versus Noradrenaline as Initial therapy in Septic sHock (VANISH) trial is a multicentre prospective, factorial $(2 \times 2)$, randomised, double-blind, placebo-controlled trial. The trial is sponsored by Imperial College London and coordinated by the Imperial Clinical Trials Unit. The trial will be conducted in accordance with the principles of the Declaration of Helsinki and Good Clinical Practice. It is registered on the European Union Drug Regulating Authorities Clinical Trials database (EudraCT 2011-005363-24) and the International Standardised Randomised Controlled Trial Registry (ISRCTN20769191). The study is jointly funded by the National Institute for Health Research (NIHR) under its Research for Patient Benefit Programme and the NIHR Clinician Scientist fellowship award scheme.

The primary objectives of this trial are:

1. To test whether vasopressin reduces renal dysfunction compared with noradrenaline when used as the initial vasopressor in the management of adult patients who have septic shock.

2. To test whether there is an interaction between vasopressin and corticosteroids when used in the management of septic shock.

The secondary objectives are to assess whether vasopressin improves other secondary outcomes (need for dialysis, survival rates, other organ failures and resource usage) compared with noradrenaline in the management of septic shock in adult patients.

\section{Setting}

The trial will be conducted in multiple general adult ICUs within the UK and will recruit 412 patients. Patients will be randomised to receive vasopressin (0$0.06 \mathrm{U} / \mathrm{min})$ or noradrenaline $(0-12 \mu \mathrm{g} / \mathrm{min})$ as the initial vasopressor therapy to maintain mean arterial blood pressure after adequate fluid resuscitation. This first study drug will continue as a continuous intravenous infusion until the septic shock has resolved and the patient no longer requires vasopressor support.

If maximum doses of the first study drug are reached ( $4.5 \mathrm{~mL} / \mathrm{h}$ of the blinded infusion), the patient will be treated with the second study drug (hydrocortisone or placebo), before additional clinically indicated vasopressors/inotropes are prescribed (see figure 1).

\section{Subject selection}

Inclusion criteria

The target population is adult patients who require vasopressors for the management of sepsis despite fluid resuscitation.

Inclusion criteria will use the internationally established consensus definitions of sepsis. ${ }^{4}$ In brief:

- Fulfil 2/4 of the criteria of the systemic inflammatory response syndrome (SIRS) due to known or suspected infection within the previous $24 \mathrm{~h}$. The SIRS criteria are:
1. Fever $\left(>38^{\circ} \mathrm{C}\right)$ or hypothermia $\left(<36^{\circ} \mathrm{C}\right)$,

2. Tachycardia (heart rate $>90 \mathrm{bpm})$,

3. Tachypnoea (respiratory rate $>20$ breaths/min or $\mathrm{paCO}_{2}<4.3 \mathrm{kPa}$ ) or need for mechanical ventilation,

4. Abnormal leucocyte count $\left(>12000\right.$ cells $/ \mathrm{mm}^{3},<4000$ cells $/ \mathrm{mm}^{3}$ or $>10 \%$ immature (band) forms).

- Hypotension despite adequate intravenous fluid resuscitation (suggested minimum of $1 \mathrm{~L}$ in the previous $4 \mathrm{~h}$, some patients may need considerably more).

\section{Exclusion criteria}

- Patient has received a continuous infusion of vasopressors previously during this ICU admission (other than vasopressors used as emergency treatment (for less than $6 \mathrm{~h}$ ) to stabilise the patient during this episode). Vasopressors include noradrenaline, epinephrine, vasopressin, dopamine, metaraminol, phenylephrine and (intermittent) terlipressin.

- Regular systemic corticosteroid therapy within the previous 3 months (this does not include inhaled steroid therapy).

- Known adrenal dysfunction/insufficiency.

- End-stage renal failure (ie, requiring long-term dialysis).

- Physician and team are not committed to full active care.

- Patient is known to be pregnant.

- Patient has known acute mesenteric ischaemia.

- Patient is known to have Raynaud's phenomenon, systemic sclerosis or other vasospastic diseases.

- Patient has been enrolled in another clinical trial of an investigational medicinal product within 30 days or is enrolled in another interventional study that might interact with the study drugs.

- Patient has a history of anaphylaxis or hypersensitivity to any study drug.

\section{Screening}

All patients who are clinically judged to have septic shock will be screened against the inclusion and exclusion criteria to be eligible for the study.

\section{Randomisation}

Randomisation (with concealed allocation) will be by pre-prepared computer-generated randomised number and will use the InForm (Oracle Corp, California, USA) online system. Randomisation will be stratified by ICU and will occur on a 1:1 basis in permuted blocks.

\section{Interventions}

The four treatment arms will be:

1. Vasopressin+hydrocortisone;

2. Vasopressin+placebo;

3. Noradrenaline+hydrocortisone;

4. Noradrenaline+placebo.

Study drug 1 will be vasopressin $(0-0.06 \mathrm{U} / \mathrm{min})$ or noradrenaline $(0-12 \mu \mathrm{g} / \mathrm{min})$ and this will be used as the first-line vasopressor for the management of septic shock. 


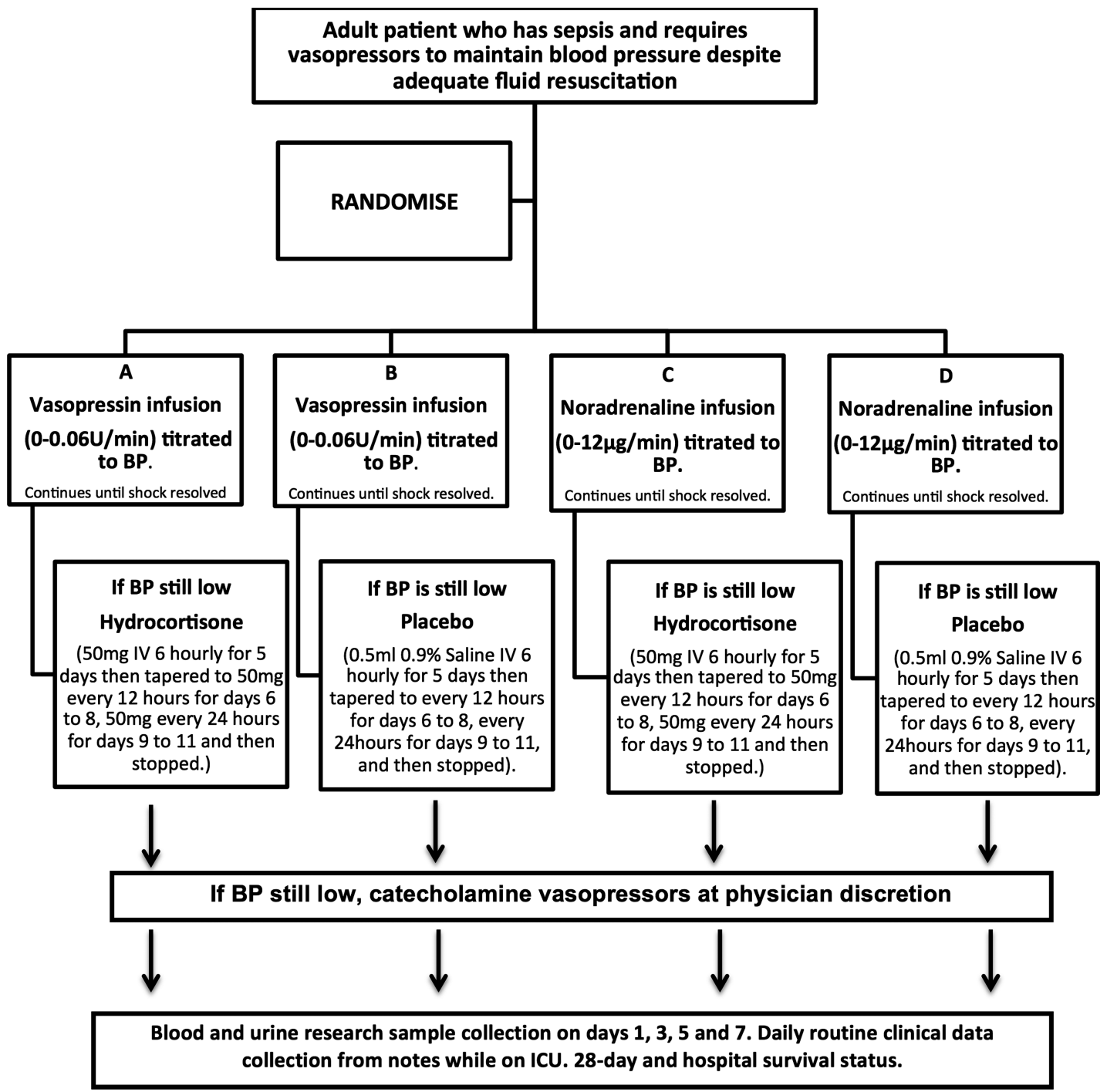

Figure 1 Patient flow. BP, blood pressure; IV, intravenous.

After study inclusion and randomisation, study drug 1 will be given via continuous infusion through a central intravenous line and titrated by the bedside nurse to achieve and maintain a target mean arterial pressure $(65-75 \mathrm{~mm} \mathrm{Hg})$. The treating physician may alter the target mean arterial pressure if clinically indicated.

All study drugs will be supplied to the ICU as specific research study drugs and they will be stored in separate research stores (eg, locked boxes in ICU). All drugs will be drawn up by the bedside critical care nurse. Blinding of all clinical staff, patients and researchers will be maintained through the use of overlabelling on the body and neck of normal drug ampoules, and matching placebo ampoules will be manufactured by Sharp Clinical Services (Powys, Wales).

Study drug 1 will come as three ampoules: one small ampoule $(2 \mathrm{~mL})$ and two large ampoules $(4 \mathrm{~mL})$; one size of ampoule will contain active drug (vasopressin or noradrenaline) and one will contain saline. All three ampoules (total $10 \mathrm{~mL}$ ) will be added to $40 \mathrm{~mL}$ of $5 \%$ dextrose into a syringe. The infusion will be started at $1 \mathrm{~mL} / \mathrm{h}$ (equal to vasopressin $0.013 \mathrm{U} / \mathrm{min}$ or noradrenaline $2.66 \mu \mathrm{g} / \mathrm{min}$ ) and increased to a maximum of $4.5 \mathrm{~mL} / \mathrm{h}$ (equal to vasopressin $0.06 \mathrm{U} / \mathrm{min}$ or noradrenaline $12 \mu \mathrm{g} / \mathrm{min})$, as clinically indicated to achieve/maintain the target mean arterial pressure.

If the maximum limit of study drug 1 is reached $(4.5 \mathrm{~mL} / \mathrm{h})$, then study drug 2 will be administered. This will be either hydrocortisone phosphate $(50 \mathrm{mg})$ or placebo ( $0.9 \%$ saline), and $0.5 \mathrm{~mL}$ of study drug 2 will be administered by intravenous injection six-hourly. This study drug will be administered for 5 days and then tapered to $0.5 \mathrm{~mL}$ every $12 \mathrm{~h}$ for days $6-8,0.5 \mathrm{~mL}$ every $24 \mathrm{~h}$ for days 9-11, and then stopped. If the septic shock has reversed (ie, all vasopressin and catecholamine infusion stopped) before these timescales, then the physician may taper study drug 2 more quickly.

If additional vasopressors are required to maintain blood pressure after the maximum rate of study drug 1 is reached $(4.5 \mathrm{~mL} / \mathrm{h})$ and the first dose of study drug 2 
(hydrocortisone or $0.9 \%$ saline) has been administered, then the treating physician may start any catecholamine vasopressor as clinically indicated (no additional vasopressin or vasopressin analogue is allowed).

The study drugs should not be started until the treating physician is confident that adequate fluid resuscitation has been achieved. Adequate fluid resuscitation should be achieved using repeated fluid challenges. Examples of appropriate targets include any or all of the following:

- Central venous pressure $\geq 8 \mathrm{~mm} \mathrm{Hg}(\geq 12 \mathrm{~mm} \mathrm{Hg}$ in mechanically ventilated patients);

- Urine output $>0.5 \mathrm{~mL} / \mathrm{kg} / \mathrm{h}$;

- $\mathrm{ScvO}_{2} \geq 70 \%$;

- Good peripheral perfusion on clinical examination;

- Other measures of cardiac output/flow.

During the study drug administration and especially during the first $6 \mathrm{~h}$, patients must be repeatedly reassessed to ensure adequate fluid resuscitation using any or all of the targets above.

Study drug 1 infusion will continue until the septic shock has resolved. When a patient is recovering from septic shock, any open-label catecholamine vasopressor infusions will be weaned first, followed by study drug 1. The rate of weaning of the drugs should be as clinically indicated to maintain the target mean arterial pressure. Once study drug 1 has stopped if vasopressors are required within $24 \mathrm{~h}$, study drug 1 should be restarted. If more than $24 \mathrm{~h}$ have elapsed and vasopressors are required again these could be with any open-label vasopressors at the treating physician's discretion. Other management of septic shock, including use of inotropes (eg, dobutamine), will be at the treating physician's discretion, based on the international 'Surviving Sepsis' guidelines. ${ }^{17}$ High-volume haemofiltration for the management of sepsis (ie, renal replacement therapy not to treat kidney failure) should not be used.

\section{Emergency treatment}

Study drug 1 should be given as the initial vasopressor to treat septic shock. However, in an emergency situation, the patient's blood pressure should be stabilised using any clinically indicated vasopressors and/or inotropes. As soon as the clinical situation has stabilised, these other vasopressors should be replaced by study drug 1 according to the study protocol and no later than $6 \mathrm{~h}$. Use of emergency vasopressors in this situation will not be an exclusion criteria or protocol violation.

\section{Rescue steroid therapy}

If patients are receiving study drugs 1 and 2 and are not responding to open-label catecholamine vasopressors (ie, life-threatening hypotension), then they may be administered intravenous hydrocortisone therapy as rescue therapy. This should not occur until the treating physician has reassessed fluid resuscitation status and a reasonable dose of catecholamine has been given an opportunity to increase the blood pressure (suggested $\geq 0.5 \mu \mathrm{g} / \mathrm{kg} / \mathrm{min}$ of noradrenaline or equivalent). In this situation, study drug 2 should be stopped and open-label steroid given as per the treating physician's prescription. Study drug 1 should continue.

Similarly, if after inclusion, systemic steroids are required to treat another medical condition (ie, not septic shock) then study drug 2 should be stopped and the necessary steroid prescribed. Study drug 1 should continue. Inhaled steroids can be prescribed as clinically indicated at any time. All other drugs (other than vasopressors) should be prescribed as clinically indicated.

\section{Unblinding}

It is unlikely that there would be a need to unblind the study drugs. The vasopressor infusions have very short half-lives, and therefore if there was an $\mathrm{AE}$, the drug could be stopped and any effects would wear off in minutes. However, emergency envelopes will be supplied to each hospital pharmacy to allow emergency unblinding if needed.

The local investigators should aim to discuss the need for unblinding with the trial coordinator or chief investigator beforehand.

\section{Laboratory evaluations}

Blood and urine sampling will be carried out in select centres. Blood, $25 \mathrm{~mL}$ for plasma, serum, RNA and DNA samples and urine $(10 \mathrm{~mL})$ will be collected on the day of inclusion and days 3,5 and 7 (see table 1). The plasma samples will be collected in EDTA and spun within $15 \mathrm{~min}$; serum samples will be collected in plain tubes and allowed to clot for 30 min before separation and RNA samples will be collected in PAXgene blood RNA tubes (PreAnalytiX GmbH, Switzerland). All samples will be frozen at $-80^{\circ} \mathrm{C}$ within $1 \mathrm{~h}$ and sent in batches to the coordinating centre for storage and later analysis. Samples will be kept beyond the end of the trial and stored in accordance with the Human Tissue Act.

\section{Data collection and management}

Participants will be followed up daily while in the ICU and to ascertain survival status at 28 days postrecruitment and hospital discharge. Routinely collected clinical data (cardiovascular, respiratory and renal physiological variables as well as haematological, biochemical and microbiological blood test results) will be recorded on a daily basis during this time and entered directly by local staff onto a trial specific electronic case report form (eCRF), InForm. All personal identifiable data, including screened patients, will be kept securely in the local site files and will not be uploaded to the main trial database.

The eCRF database generates automatic alerts for missing, invalid or data which does not conform to the rules established for that data type. There is an electronic audit trial for all data changes. In addition, the central coordinating site will visit local recruiting sites to ensure compliance with the protocol, Good Clinical 
Table 1 Visit schedule

\begin{tabular}{|c|c|c|c|c|c|c|c|c|}
\hline Visit & $\begin{array}{l}\text { Day } \\
1\end{array}$ & $\begin{array}{l}\text { Day } \\
2\end{array}$ & $\begin{array}{l}\text { Day } \\
3\end{array}$ & $\begin{array}{l}\text { Day } \\
4\end{array}$ & $\begin{array}{l}\text { Day } \\
5\end{array}$ & $\begin{array}{l}\text { Day } \\
6\end{array}$ & $\begin{array}{l}\text { Day } \\
7\end{array}$ & $\begin{array}{l}\text { Day } \\
8-28\end{array}$ \\
\hline Screening & \multicolumn{8}{|c|}{$\mathrm{X}$} \\
\hline $\begin{array}{l}\text { Informed consent (patient consent/PerLR/ProLR/retrospective } \\
\text { patient information) }\end{array}$ & \multicolumn{8}{|c|}{$\begin{array}{l}\text { Retrospective consent (PerLR/ProLR) will be obtained at the } \\
\text { first available opportunity. Retrospective patient consent will } \\
\text { be obtained when the patient has recovered }\end{array}$} \\
\hline Inclusion/exclusion criteria & \multicolumn{8}{|c|}{$\mathrm{X}$} \\
\hline Randomisation & \multicolumn{8}{|c|}{$\mathrm{x}$} \\
\hline Study drug administration (study drug 1 and 2) & \multicolumn{8}{|c|}{$\begin{array}{l}\text { Study drug } 1 \text { (vasopressin/noradrenaline) continued until } \\
\text { shock resolved. If BP was still low, study drug } 2 \\
\text { (hydrocortisone/placebo) administered as described in this } \\
\text { protocol }\end{array}$} \\
\hline \multicolumn{9}{|c|}{ 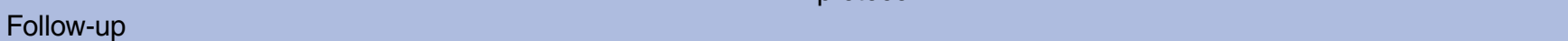 } \\
\hline Blood and urine sampling & $X$ & & $\mathrm{X}$ & & $\mathrm{X}$ & & $X$ & \\
\hline Daily collection of clinical data & $\mathrm{x}$ & $\mathrm{X}$ & $\mathrm{X}$ & $\mathrm{X}$ & $\mathrm{X}$ & $\mathrm{X}$ & $\mathrm{X}$ & $\mathrm{X}$ \\
\hline Final visit & \multicolumn{8}{|c|}{ On the day of discharge from the hospital } \\
\hline
\end{tabular}

Practice and local regulatory compliance as well as source data verification.

\section{Primary outcome}

The primary outcome of the trial will be the difference in renal failure-free days (the number of days alive and free of renal failure) during the 28 days after randomisation between treatment groups. Renal failure will be defined by the Acute Kidney Injury Network (AKIN) group stage 3 definition ${ }^{28}$ :

- Increase in serum creatinine to $>300 \%$ (>threefold) from baseline;

- Or serum creatinine $\geq 354 \mu \mathrm{mol} / \mathrm{L}$ with an acute rise of at least $44 \mu \mathrm{mol} / \mathrm{L}$;

- Or initiation of renal replacement therapy;

- Or a urine output of $<0.3 \mathrm{~mL} / \mathrm{kg} / \mathrm{h} \geq 24 \mathrm{~h}$;

- Or anuria $\geq 12 \mathrm{~h}$.

\section{Secondary outcomes}

Secondary outcomes will include:

- Rates and duration of renal replacement therapy;

- Length of renal failure in survivors and non-survivors;

- 28-day, ICU and hospital mortality rates;

- Organ failure-free days in the first 28 days, assessed using the Serial Organ Failure Assessment (SOFA) score $;^{29}$

- Organ support data assessed using the standard National Health Service Healthcare Resource Groups;

- Blood and urinary biomarkers of renal function and inflammation (for subsequent analyses).

\section{ETHICS}

The trial protocol and participant information sheets have been reviewed by the Oxford A independent research ethics committee and given a favourable opinion (12/SC/0014). It has clinical trials approval from the Medicines and Healthcare Products Regulatory
Authority (MHRA). Any substantial protocol amendments will be sent to the ethics committee and MHRA for approval as per the standard regulatory requirements. All sites will then be informed of the amendments.

\section{Informed consent}

In most cases, it will not be possible to obtain prospective consent from the patient at the time of enrolment. This is due to the fact that many patients will have a reduced level of consciousness due to their illness or due to sedative medication used as part of their treatment. As all the study drugs are already routinely used in the management of septic shock, there is minimal extra risk from participation in this study. As septic shock is a medical emergency and the fact that delays in administering the study drugs may affect patient outcome, the patient will be treated and samples collected in the emergency situation without prospective consent. No analysis of data or samples will occur until retrospective consent is obtained. At the first available opportunity, once the clinical condition has stabilised, retrospective consent should be sought. This process was used in the pilot/feasibility study ${ }^{27}$ and was found to be acceptable to clinicians, patients and their families.

\section{Patient consent}

If possible, informed consent will be obtained from the patient. The patient will be informed about the trial by the responsible clinician or a member of the research team and given a copy of the patient information sheet (PIS). Informed patients will be given an adequate amount of time to consider their participation in the trial. If the patient decides to participate in the trial, they will be asked to sign the patient consent form which will then be countersigned by the responsible clinician. 


\section{Personal legal representative consent}

If the patient is unable to give consent, informed consent will be sought from the patient's 'Personal Legal Representative' (PerLR) who may be a relative, partner or close friend. The PerLR will be informed about the trial by the responsible clinician or a member of the research team and provided with a copy of the covering statement for PerLR with an attached PerLR information sheet and asked to give an opinion as to whether the patient would object to taking part in such medical research. If the PerLR decides that the patient would have no objection to participating in the trial, they will be asked to sign the PerLR consent form which will then be countersigned by the responsible clinician.

\section{Professional legal representative consent}

If the patient is unable to give informed consent and no PerLR is immediately available, a doctor who is not connected with the conduct of the trial may act as a Professional Legal Representative (ProLR). The doctor will be informed about the trial by the responsible clinician or a member of the research team and given a copy of the PIS. If the doctor decides that the patient is suitable for entry into the trial, they will be asked to sign the ProLR consent form.

\section{Retrospective patient information}

Patients for whom consent is given by a PerLR or ProLR will be informed of their participation in the trial by the responsible clinician or a member of the research team once they regain capacity to understand the details of the trial. The responsible clinician will discuss the study with the patient and the patient will be given a copy of the PIS to keep. The patient will be asked for consent to continue participation in the trial and to sign the consent form. If the patient does not want to continue participation in the study, they will be given the choice of having already collected data and samples excluded from the final analysis. All participants are free to withdraw at any time from the protocol treatment without giving reasons and without prejudicing further treatment.

If the patient dies before consent is obtained, retrospective consent should be sought from the PerLR (or ProLR, if no PerLR can be identified) for inclusion and collection of data.

\section{Monitoring and pharmacovigilance}

Given that the trial is being conducted on critically ill patients, a significant number of patients are likely to experience AEs. Events that are in keeping with the patient's clinical condition will not be reported as AEs. The following clinical outcomes from sepsis will not be recorded as AEs unless the local investigator deems the event to be related to the study drug or the protocoldeath, cardiovascular failure, respiratory failure, hepatic failure, renal failure and haematological failure. Specific information about the occurrence of a myocardial infarction or acute coronary syndrome, life-threatening arrhythmias, mesenteric ischaemia, digital ischaemia and secondary infection will be recorded in the case report form. All AEs will be assessed for likely relationship to the study drugs. All SAEs or a suspected unexpected serious adverse reaction will be reported to the trial coordination centre within $24 \mathrm{~h}$ and to the relevant authorities in accordance with current regulations.

Imperial College London, the sponsor of the trial has civil liability insurance, which covers this study in all participating centres. Imperial College London also holds negligent harm and non-negligent harm insurance policies which apply to this study.

\section{Statistical analysis plan}

The main analysis will be carried out on an intention-to-treat basis. However, as not all patients will require study drug 2, analysis will also be carried out on an 'as treated' basis and sensitivity analysis performed. Every effort will be made to minimise missing baseline and outcome data in this trial, but prior to data analysis, the level and pattern of the missing data will be established and the likely causes of any missingness investigated. If necessary, multiple imputation ${ }^{30}{ }^{31}$ or Bayesian methods for missing data ${ }^{32}$ will be used as appropriate to address any issues arising.

The primary outcome, the number of days alive and free of renal failure, is not normally distributed and has a large spike in frequency at 28 days, the point at which the measure is truncated. Therefore, the data will be reported as a combination of two summary measures:

- The proportion of patients who survive and have no renal failure during the 28 days after randomisation;

- The median number of days alive and free of renal failure for patients who do not survive and/or experience some renal failure during the 28 days after randomisation.

For the main analysis, we will test for a difference between the distribution of days alive and free of renal failure for participants randomised to vasopressin compared with those randomised to noradrenaline using a Mann-Whitney U test. A Markov model with three states (alive and no renal failure, alive with renal failure and death) and including indicator variables for vasopressin, corticosteroids and vasopressin $\times$ corticosteroids (interaction term) will be fitted to assess the possible interaction between vasopressin and corticosteroids, and estimate how much of any increase in days alive and free of renal failure can be attributed to preventing renal failure and how much to preventing death.

The secondary outcomes will be analysed using appropriate regression models to compare the four treatment groups. Comparisons of vasopressin versus noradrenaline and corticosteroids versus placebo will also be presented, provided the strength of any interaction does not render these 'at the margins' analyses unreliable.

The sample size was chosen to provide $80 \%$ power to detect a $20-25 \%$ relative reduction of risk of developing renal failure if treated with vasopressin compared with 
noradrenaline, assuming an overall incidence of acute renal failure of $30-50 \%$ and a significance level of 0.05 . The calculations were based on simulation, assuming a Mann-Whitney $U$ test is used to analyse the primary outcome. The 412 patients allow for a 3\% withdrawal of consent in line with previous critical care studies within the UK. ${ }^{33}$ Owing to the emergency nature of the trial and the rapid timescales required for inclusion, as in the pilot trial, ${ }^{27}$ it is possible that new clinical information may become apparent after randomisation but before starting the study drug. If the patient is discovered to meet any exclusion criteria or not meet the inclusion criteria before the study drug has started, they will not be included in the trial and its analysis. These patients will be replaced.

\section{End of trial}

The trial will end once 412 patients have been enrolled, and the last patient has reached day 28 postenrolment or at hospital discharge, whichever is later, or earlier if mandated by the Trial Steering Committee (TSC) or sponsor.

\section{Trial monitoring and oversight}

The TSC with an independent chair, independent members including patient and public representatives will be responsible for overseeing the progress of the trial and will meet six-monthly. The independent Data Monitoring and Ethics Committee (DMEC) will meet six-monthly to review ongoing recruitment, protocol compliance, clinical outcome and $\mathrm{AE}$ data. They will provide the TSC with written reports to advise about ongoing trial conduct.

\section{DISSEMINATION}

Results will be presented at scientific meetings and published in peer-reviewed journals. All publications and presentations relating to the study will be authorised by the Trial Management Group. Authorship will be determined according to the internationally agreed criteria for authorship. In addition, a lay summary will be available on the websites of the Imperial Clinical Trials Unit and the Intensive Care Foundation. Investigators who would like access to the trial data set are encouraged to submit a brief application, outlining rationale and analytical plan, to the trial management group for approval for additional studies.

\section{Trials status}

Recruitment is ongoing. The first patient was recruited in February 2013 and we expect recruitment to be complete in May 2015.

\section{Author affiliations}

${ }^{1}$ Section of Anaesthetics, Pain Medicine and Intensive Care, Department of Surgery and Cancer, Imperial College London, London, UK

${ }^{2}$ Centre for Perioperative Medicine and Critical Care Research, Imperial College Healthcare NHS Trust, London, UK
${ }^{3}$ Imperial Clinical Trials Unit, School of Public Health, Faculty of Medicine, Imperial College London, London, UK

${ }^{4}$ Warwick Clinical Trials Unit, University of Warwick, Coventry, UK

${ }^{5}$ Heart of England NHS Foundation Trust, Birmingham, UK

Acknowledgements The authors are grateful for the infrastructure provided by the Critical Care Specialty Group of the Comprehensive Clinical Research Network. The study is included in the NIHR Clinical Research Network (NIHR CRN) portfolio (ID 12985)

Contributors ACG conceived the initial trial concept and designed the protocol. AJM, GDP, DA and SJB helped develop the trial design and protocol. AJM and DA carried out the power calculations and wrote the statistical analytical plan. ACG is the chief investigator and takes overall responsibility for all aspects of trial design, the protocol and trial conduct. All authors read and approved the final manuscript.

Funding The trial is funded by the National Institute for Health Research (NIHR) under its Research for Patient Benefit programme (PB-PG-061022350). It is also supported by the Intensive Care Foundation and the NIHR Comprehensive Biomedical Research Centre based at Imperial College Healthcare NHS Trust and Imperial College London.

Competing interests ACG is an NIHR Clinician Scientist Fellowship award holder. ACG and GDP are directors of Research of the Intensive Care Foundation, and SJB was previously the chair of the Foundation. ACG is named as an inventor on a University of British Columbia patent application related to vasopressin. DA is a NIHR senior investigator.

Ethics approval National Research Ethics Committee Oxford A (12/SC/0014).

Provenance and peer review Not commissioned; peer reviewed for ethical and funding approval prior to submission.

Disclaimer This paper presents independent research funded by the NIHR. The views expressed are those of the authors and not necessarily those of the NHS, the NIHR or the Department of Health.

Open Access This is an Open Access article distributed in accordance with the Creative Commons Attribution Non Commercial (CC BY-NC 4.0) license, which permits others to distribute, remix, adapt, build upon this work noncommercially, and license their derivative works on different terms, provided the original work is properly cited and the use is non-commercial. See: http:// creativecommons.org/licenses/by-nc/4.0/

\section{REFERENCES}

1. Hoyert DL, Kung HC, Smith BL. Deaths: preliminary data for 2003 Natl Vital Stat Rep 2005;53:1-48.

2. Harrison DA, Welch CA, Eddleston JM. The epidemiology of severe sepsis in England, Wales and Northern Ireland, 1996 to 2004: secondary analysis of a high quality clinical database, the ICNARC Case Mix Programme Database. Crit Care 2006;10:R42.

3. Vincent JL, Sakr Y, Sprung CL, et al. Sepsis in European intensive care units: results of the SOAP study. Crit Care Med 2006;34:344-53.

4. [No authors listed]. American College of Chest Physicians/Society of Critical Care Medicine Consensus Conference: definitions for sepsis and organ failure and guidelines for the use of innovative therapies in sepsis. Crit Care Med 1992;20:864-74.

5. Hayes MA, Timmins AC, Yau EH, et al. Elevation of systemic oxygen delivery in the treatment of critically ill patients. $N$ Engl J Med 1994;330:1717-22.

6. Landry DW, Levin HR, Gallant EM, et al. Vasopressin deficiency contributes to the vasodilation of septic shock. Circulation 1997;95:1122-5.

7. Patel BM, Chittock DR, Russell JA, et al. Beneficial effects of short-term vasopressin infusion during severe septic shock. Anesthesiology 2002;96:576-82.

8. Edwards RM, Trizna W, Kinter LB. Renal microvascular effects of vasopressin and vasopressin antagonists. Am J Physiol 1989;256(2 Pt 2):F274-8.

9. Bomzon L, Rosendorff C. Renovascular resistance and noradrenaline. Am J Physiol 1975;229:1649-53.

10. Thibonnier M, Conarty DM, Preston JA, et al. Human vascular endothelial cells express oxytocin receptors. Endocrinology 1999;140:1301-9. 
11. Jin HK, Chen YF, Yang $\mathrm{RH}$, et al. Vasopressin lowers pulmonary artery pressure in hypoxic rats by releasing atrial natriuretic peptide. Am J Med Sci 1989;298:227-36.

12. Okamura $\mathrm{T}$, Ayajiki $\mathrm{K}$, Fujioka $\mathrm{H}$, et al. Mechanisms underlying arginine vasopressin-induced relaxation in monkey isolated coronary arteries. J Hypertens 1999;17:673-8.

13. Suzuki $\mathrm{Y}$, Satoh $\mathrm{S}$, Oyama $\mathrm{H}$, et al. Regional differences in the vasodilator response to vasopressin in canine cerebral arteries in vivo. Stroke 1993;24:1049-53.

14. Oyama H, Suzuki $\mathrm{Y}$, Satoh $\mathrm{S}$, et al. Role of nitric oxide in the cerebral vasodilatory responses to vasopressin and oxytocin in dogs. J Cereb Blood Flow Metab 1993;13:285-90.

15. Serpa Neto A, Nassar APJ, Cardoso SO, et al. Vasopressin and terlipressin in adult vasodilatory shock: a systematic review and meta-analysis of nine randomized controlled trials. Crit Care 2012;16:R154.

16. Polito A, Parisini E, Ricci Z, et al. Vasopressin for treatment of vasodilatory shock: an ESICM systematic review and meta-analysis. Intensive Care Med 2012;38:9-19.

17. Dellinger RP, Levy MM, Carlet JM, et al. Surviving Sepsis Campaign: international guidelines for management of severe sepsis and septic shock: 2008. Intensive Care Med 2008;34:17-60.

18. Russell JA, Walley KR, Singer J, et al. Vasopressin versus noradrenaline infusion in patients with septic shock. $N$ Engl J Med 2008;358:877-87.

19. Mehta S, Granton J, Gordon AC, et al. Cardiac ischemia in patients with septic shock randomized to vasopressin or noradrenaline. Crit Care 2013;17:R117.

20. Gordon AC, Wang N, Walley KR, et al. The cardiopulmonary effects of vasopressin compared with noradrenaline in septic shock. Chest 2012;142:593-605.

21. Gordon AC, Russell JA, Walley KR, et al. The effects of vasopressin on acute kidney injury in septic shock. Intensive Care Med 2010;36:83-91.
22. Parrillo JE. Septic shock-vasopressin, noradrenaline, and urgency. N Engl J Med 2008;358:954-6.

23. Russell JA, Walley KR, Gordon AC, et al. Interaction of vasopressin infusion, corticosteroid treatment, and mortality of septic shock. Crit Care Med 2009;37:811-18

24. Sprung CL, Annane D, Keh D, et al. Hydrocortisone therapy for patients with septic shock. N Engl J Med 2008;358:111-24.

25. Annane D, Bellissant E, Bollaert PE, et al. Corticosteroids in the treatment of severe sepsis and septic shock in adults: a systematic review. JAMA 2009;301:2362-75

26. Minneci PC, Deans KJ, Eichacker PQ, et al. The effects of steroids during sepsis depend on dose and severity of illness: an updated meta-analysis. Clin Microbiol Infect 2009;15:308-18.

27. Gordon AC, Mason AJ, Perkins GD, et al. The interaction of vasopressin and corticosteroids in septic shock: a pilot randomized controlled trial. Crit Care Med 2014;42:1325-33.

28. Mehta RL, Kellum JA, Shah SV, et al. Acute Kidney Injury Network: report of an initiative to improve outcomes in acute kidney injury. Crit Care 2007;11:R31.

29. Vincent JL, Moreno R, Takala J, et al. The SOFA (Sepsis-related Organ Failure Assessment) score to describe organ dysfunction/ failure. On behalf of the Working Group on Sepsis-Related Problems of the European Society of Intensive Care Medicine. Intensive Care Med 1996;22:707-10

30. Sterne JA, White IR, Carlin JB, et al. Multiple imputation for missing data in epidemiological and clinical research: potential and pitfalls. BMJ 2009;338:b2393.

31. Kenward MG, Carpenter J. Multiple imputation: current perspectives. Stat Methods Med Res 2007;16:199-218.

32. Daniels MJ, Hogan JW, eds. Missing data in longitudinal studies: strategies for Bayesian modeling and sensitivity analysis. 1st edn. Chapman and Hall, 2008

33. Harvey SE, Elbourne D, Ashcroft J, et al. Informed consent in clinical trials in critical care: experience from the PAC-Man Study. Intensive Care Med 2006;32:2020-5. 